\title{
Abscisic Acid Interacts Antagonistically with Salicylic Acid Signaling Pathway in Rice-Magnaporthe grisea Interaction
}

\author{
Chang-Jie Jiang, ${ }^{1}$ Masaki Shimono,, ${ }^{1}$ Shoji Sugano, ${ }^{1}$ Mikiko Kojima, ${ }^{2}$ Katsumi Yazawa, ${ }^{1}$ \\ Riichiro Yoshida, ${ }^{1}$ Haruhiko Inoue, ${ }^{1}$ Nagao Hayashi, ${ }^{1}$ Hitoshi Sakakibara, ${ }^{2}$ and Hiroshi Takatsuji ${ }^{1}$ \\ ${ }^{1}$ Plant Disease Resistance Research Unit, National Institute of Agrobiological Sciences, Kannondai 2-1-2, Tsukuba, \\ 305-8602 Japan; '²Plant Productivity Systems Research Group, RIKEN Plant Science Center, Suehiro 1-7-22, Tsurumi, \\ Yokohama 230-0045, Japan
}

Submitted 28 September 2009. Accepted 12 February 2010.

Plant hormones play pivotal signaling roles in plant-pathogen interactions. Here, we report characterization of an antagonistic interaction of abscisic acid (ABA) with salicylic acid (SA) signaling pathways in the rice-Magnaporthe grisea interaction. Exogenous application of ABA drastically compromised the rice resistance to both compatible and incompatible $M$. grisea strains, indicating that $\mathrm{ABA}$ negatively regulates both basal and resistance gene-mediated blast resistance. ABA markedly suppressed the transcriptional upregulation of WRKY45 and OSNPR1, the two key components of the SA signaling pathway in rice, induced by SA or benzothiadiazole or by blast infection. Overexpression of $O S N P R 1$ or WRKY45 largely negated the enhancement of blast susceptibility by ABA, suggesting that ABA acts upstream of WRKY45 and OsNPR1 in the rice $S A$ pathway. $A B A$-responsive genes were induced during blast infection in a pattern reciprocal to those of $W R K Y 45$ and $O S P R 1 b$ in the compatible rice-blast interaction but only marginally in the incompatible one. These results suggest that the balance of $\mathrm{SA}$ and $\mathrm{ABA}$ signaling is an important determinant for the outcome of the rice- $M$. grisea interaction. ABA was detected in hyphae and conidia of $M$. grisea as well as in culture media, implying that blast-fungus-derived ABA could play a role in triggering ABA signaling at host infection sites.

Plants have evolved a battery of defense mechanisms to protect themselves from microbial pathogen attacks, in which plant hormones salicylic acid (SA), jasmonic acid (JA), ethylene (ET), and abscisic acid (ABA) play central signaling roles (Grant and Jones 2009; Lopez et al. 2008; Robert-Seilaniantz et al. 2007). Each of these hormones generates and transmits a distinct defense signal, while cooperative or antagonistic crosstalks between them are emerging as pivotal for outcomes of disease resistance. Microbial pathogens, on the other hand, have also evolved virulence mechanisms to avoid plant defenses by manipulating hormone balances (Asselbergh et al. 2008 b; Lopez et al. 2008). The most well-known example of such interaction is the perturbation of the SA/JA balance in

Corresponding author: Hiroshi Takatsuji; Telephone and Fax: +81-29838-8383; E-mail: takatsuh@ affrc.go.jp

* The $\boldsymbol{e}$-Xtra logo stands for "electronic extra" and indicates that five supplementary figures and one supplementary table are available online.
Arabidopsis by a biotrophic bacterial pathogen Pseudomonas syringae pv. tomato DC3000 (DC3000) (Brooks et al. 2005; Laurie-Berry et al. 2006). SA and JA mediate defense signaling against biotrophic and necrotrophic pathogens, respectively (Loake and Grant 2007). In most cases, these two signaling pathways mutually oppose each other (Loake and Grant 2007). This antagonism between SA and JA is targeted by some $P$. syringae strains that produce the phytotoxin coronatine, a JAisoleucine analog that functionally mimics JA and suppresses SA signaling, thereby compromising host resistance to the biotrophic pathogen (Brooks et al. 2005; Laurie-Berry et al. 2006; Lopez et al. 2008). Many other fungal and bacterial pathogens have also been found to be capable of manipulating host hormone signaling pathways to their own advantage by either producing plant hormones or plant hormone-like substances by themselves or altering the hormone synthesis pathways of host plants (de Torres-Zabala et al. 2007; RobertSeilaniantz et al. 2007). Therefore, it is proposed that "successful defense likely requires that the plant attenuate any microbe-induced hormonal perturbation" (Grant and Jones 2009).

$\mathrm{SA}$ is involved in the activation of systemic acquired resistance (SAR) in many dicots such as Arabidopsis and tobacco (Durrant and Dong 2004; Vallad and Goodman 2004). A transcriptional cofactor, NPR1, plays a key role in mediating the SA signaling pathway in Arabidopsis (Dong 2004). Rice also has an SA signaling pathway that shows some similarities to that in Arabidopsis and the rice ortholog of NPR1, OsNPR1/NH1, plays a role in rice resistance to the leaf blight bacteria Xanthomonas oryzae pv. oryzae (Chern et al. 2001, 2005; Fitzgerald et al. 2004; Yuan et al. 2007). We previously identified a rice transcription factor, WRKY45, whose gene is highly, specifically induced by SA and a chemical defense inducer, benzothiadiazole (BTH). WRKY45 was also found to be a key regulator of the SA signaling pathway and involved in BTH-induced resistance to Magnaporthe grisea (Shimono et al. 2007) and X. oryzae pv. oryzae (Shimono, unpublished). Notably, the SA signaling pathway in rice was found to branch into the OsNPR1-dependent and the WRKY45-dependent pathways downstream of SA (Shimono et al. 2007), unlike the SA signaling pathway in Arabidopsis, which is primarily mediated by NPR1 (Wang et al. 2006).

ABA is an important plant hormone that plays signaling roles in diverse processes of plant growth and development as well as in plant responses to various abiotic stresses (YamaguchiShinozaki and Shinozaki 2006). Recently, ABA has also emerged as a key signaling molecule in plant-pathogen inter- 
actions (Asselbergh et al. 2008b; Mauch-Mani and Mauch 2005). Exogenous application of ABA or ABA accumulation due to genetic defects has been shown to enhance the susceptibility of various plant species to bacterial and fungal pathogens (Achuo et al. 2006; Fan et al. 2009; Henfling et al. 1980; Koga et al. 2004; Matsumoto et al. 1980; Mohr and Cahill 2003; Ward et al. 1989). Conversely, ABA-deficient mutation results in an enhanced resistance to various pathogens in tomato (Achuo et al. 2006; Asselbergh et al. 2007, 2008a; Audenaert et al. 2002) and Arabidopsis (de Torres-Zabala et al. 2009; Mohr and Cahill 2003). These mutant plants display stronger SA-dependent defense responses than do wild-type plants, suggesting that the antagonistic interaction of ABA on the SA signaling pathway underlies the negative impact of ABA on plant defense responses. This notion is also supported by a recent study that has shown antagonistic crosstalk between SA-mediated activation of SAR and ABA-mediated abiotic stress responses (Yasuda et al. 2008). Interestingly, de TorresZabala and associates (2007) have shown that $P$. syringae type III effectors (T3Es) hijack the Arabidopsis ABA biosynthetic pathway to antagonize SA levels and suppress plant basal defenses, suggesting an important role of ABA in the patho-

A
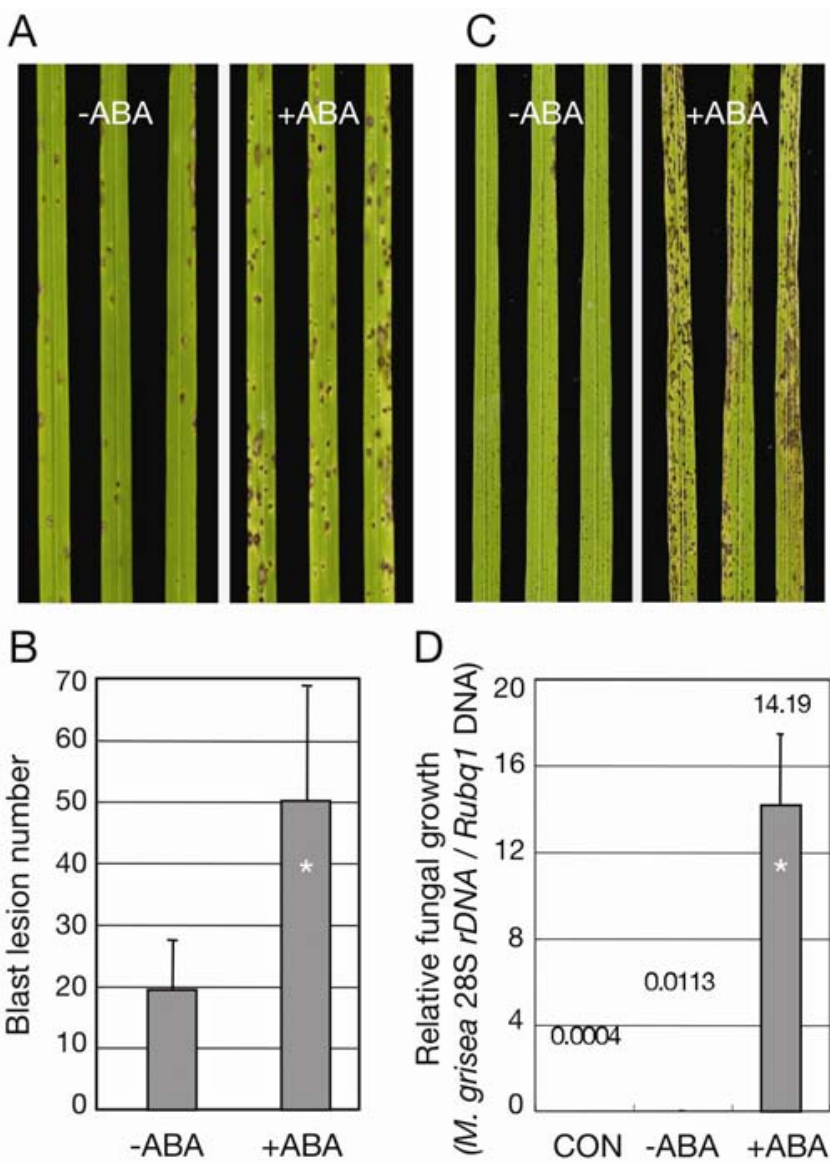

\section{$\mathrm{D}$}

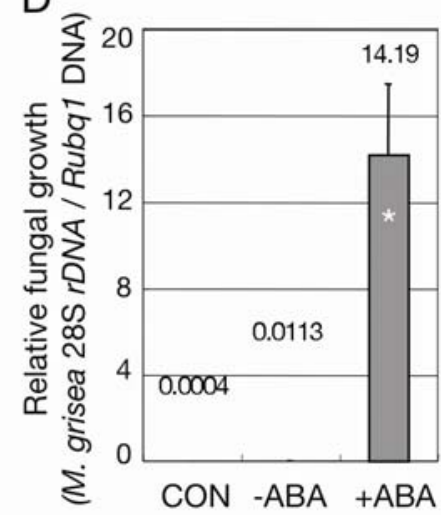

Fig. 1. Effects of exogenous abscisic acid (ABA) on the susceptibility of rice plants to compatible and incompatible blast fungus. A and $\mathbf{B}$, Compatible race 007.0 and $\mathbf{C}$ and $\mathbf{D}$, incompatible race 102.0 of Magnaporthe grisea were spray inoculated onto Nipponbare rice. A and $\mathbf{C}$, Disease symptoms at 6 days postinoculation (dpi) in fungus-inoculated rice plants after pretreatment by spraying with mock (-ABA) or $0.1 \mathrm{mM}$ ABA (+ABA) solutions. B, Average lesion numbers per $10-\mathrm{cm}$ middle regions of leaf blades ( 6 dpi) are shown with standard deviations (s.d.). D, Severity of blast disease was evaluated by the amounts of $M$. grisea genomic $28 \mathrm{~S}$ rDNA relative to rice genomic Rubql DNA (6 dpi). Mean values of three determinations with s.d. are shown. CON, leaf blades of noninoculated rice seedlings. Asterisks indicate a significant difference from the mock control $(t$ test, $P<0.01)$. genicity. On the other hand, some studies have shown positive roles of ABA in plant defense (e.g., via regulating stomatal closure [Melotto et al. 2006] and priming callose deposition [Flors et al. 2008; Ton and Mauch-Mani 2004]). Thus, ABA appears to play different roles in defense, depending upon pathosystems and infection stages. (Asselbergh et al. 2008b; Mauch-Mani and Mauch 2005).

The negative impact of ABA has also been reported in rice defenses to M. grisea (Koga et al. 2004; Matsumoto et al. 1980). Rice genes responsive to ABA and dehydration stresses were noted to be induced during infection by a compatible race of $M$. grisea (Ribot et al. 2008). Bailey and associates (2009) reported that ABA antagonistically interacts with the ET signaling pathway and, consequently, increases blast susceptibility in a compatible rice- $M$. grisea interaction. These reports support the involvement of $\mathrm{ABA}$ in rice- $M$. grisea interaction; however, the manner in which ABA signaling is generated and integrated into the rice defense pathway during rice-pathogen interactions remains largely unknown.

In the present study, we investigated the molecular mechanisms underlying the effects of ABA in enhancing the susceptibility of rice to $M$. grisea, focusing on the antagonistic interaction of ABA with the SA signaling pathway.

\section{RESULTS}

Exogenous ABA compromises rice defense to $M$. grisea.

To confirm the effect of ABA on rice blast resistance and expand the scope of the investigation, we tested the effects of ABA application on both compatible (Fig. 1A and B) and incompatible (Fig. $1 \mathrm{C}$ and D) rice- $M$. grisea interactions. Foliar spray treatment of Nipponbare rice seedlings with ABA rendered the seedlings more susceptible to a compatible $M$. grisea race (race 007.0) (Fig. 1A and B). The rice cv. Nipponbare has resistance genes Pish (Imbe and Matsumoto 1985) and Pil9 (Hayashi et al. 1998) that confer race-specific resistance to avirulent races of blast fungus 102.0 and 002.0, respectively. Inoculation with race 102.0 caused numerous small necrotic lesions in the leaves of control plants, probably due to hypersensitive response (HR) cell death (Fig. 1C), whereas race 002.0 caused fewer visible necrotic lesions (Supplementary Fig. S1A). Pretreatment by ABA caused a compromise in the resistance of Nipponbare rice to both the avirulent blast fungus races, 102.0 (Fig. 1C and D) and 002.0, giving rise to severe disease symptoms. These data indicate that ABA has a negative impact on both basal and resistance $(R)$ gene-mediated rice defense to $M$. grisea. Soil drenching with ABA resulted in similar enhancement in disease severity in a dose-dependent manner (Supplementary Fig. S2).

\section{ABA suppresses SA-induced transcriptional upregulation of WRKY45 and OsNPR1.}

In Arabidopsis, ABA has been shown to antagonize the SA signaling pathway, and this antagonism is considered responsible for the enhancement of disease susceptibility by ABA (de Torres-Zabala et al. 2007; Yasuda et al. 2008). These studies prompted us to test the effects of ABA, as well as of other hormones, on the expression of WRKY45 and OsNPRl, the two key regulators in the rice SA pathway, with each regulating one of two pathways that branch downstream of SA. WRKY45 upregulates highly specifically in response to SA and BTH; moreover, OsNPRl is also responsive to BTH (Shimono et al. 2007). Leaf blade segments were treated with SA alone or SA plus various plant hormones, and examined for the expression of WRKY45 and OsNPR1. WRKY45 expression was upregulated by SA and this upregulation was specifically and strongly suppressed by ABA (Fig. 2A). On the other hand, OsNPRl ex- 
pression was upregulated in the samples submerged in aqueous solvent (mock), indicating that mere submergence can induce OsNPRl expression in leaf blade segments (Fig. 2B). Thus, the effect of submergence obscured the effects of SA on OsNPRl expression. However, ABA was found to strongly suppress OSNPRI expression in submerged leaf blade segments to a level below that in the control sample without submergence (CON) (Fig. 2B). The induction of OsNPR1 expression in aqueous solutions may be attributed to decreased ABA levels due to submergence, because ABA becomes rapidly oxidized to phaseic acid by ABA 8'-hydroxylase in submerged rice plants (Kende et al. 1998; Saika et al. 2007). This result also implies that the basal expression of OsNPRI is also negatively regulated by ABA. The effectiveness of ABA treatment was validated by examining the induction of an ABA-responsive gene, SalT (Rabbani et al. 2003) (Figs. 2C and 3C).

To assess the relevance of the expression levels of WRKY45 and $O S N P R 1$ - which were modified by BTH or ABA treatments-with the development of blast disease, we pretreated rice seedlings with $\mathrm{BTH}, \mathrm{ABA}$, or BTH plus ABA for $24 \mathrm{~h}$ and then inoculated with compatible blast fungus (race 007.0). Both WRKY45 and OsNPR1 were induced by BTH after $24 \mathrm{~h}$ of the treatment, and the effect persisted until day 5 after blast inoculation (Fig. 3A and B). On the other hand, ABA largely offset the effects of BTH on the upregulation of WRKY45 and OsNPRl, and also decreased the basal level of OsNPRl transcripts (Fig. 3A and B). Moreover, ABA also strongly suppressed the expression of WRKY45 and OSNPRI induced by blast inoculation, which was partially alleviated by cotreatment with BTH (Fig. 3A and B). Interestingly, the ABA-responsive gene $S a l T$ was induced by blast inoculation at later time points, and this induction was enhanced by pretreatment with ABA (Fig. 3C). Meanwhile, BTH moderately suppressed the upregulation of SalT induced by blast inoculation. These results indicate that SA and ABA mutually antagonize each other's signaling pathways in rice. We also observed the antagonism between $\mathrm{SA}$ and $\mathrm{ABA}$ in the expression of rice ABA-responsive genes, $R A B 16 b$ (Yamaguchi-Shinozaki et al. 1989), OsWsil8 (Joshee et al. 1998), and SalT, after treatment of rice leaf segments with ABA or ABA plus SA (Supplementary Fig. S3). Determination of blast fungal DNA revealed that the growth of blast fungus was inversely correlated with the expression levels of WRKY45 and OSNPRI in rice plants (Fig. 3D). Thus, BTH upregulated both WRKY45 and OsNPRI and reduced fungal growth; meanwhile, $\mathrm{ABA}$ antagonized the effects of BTH on both gene expression and fungal growth. These data strongly suggest that the suppression of SA-responsive upregulation of - as well as the basal-expression of WRKY45 and OSNPRI underlies the reduction in the BTH-induced and basal resistance of rice to $M$. grisea by ABA application. It should be noted that ABA had no direct effect on the growth and morphology of M. grisea (Koga et al. 2004).

Recently, Qiu and Yu (2009) have reported that WRKY45 is constitutively expressed in various rice tissues and that ABA treatment induced WRKY45 expression. In this report, submerging whole seedlings in $100 \mu \mathrm{M}$ ABA solution induced a transient expression of WRKY45 at $1 \mathrm{~h}$. In our time-course experiment using leaf segments, however, we did not observe ABA-responsive changes in WRKY45 expression except for a small, nonspecific increase at $0.5 \mathrm{~h}$ (Supplementary Fig. S4).

\section{SA and ABA signaling are inversely activated during blast infection.}

To further investigate the interaction between SA and ABA signaling pathways during blast infection, we examined the temporal expression patterns of SA-responsive WRKY45 and ABA-responsive gene SalT, following inoculation with com- patible (007.0) or incompatible (102.0) blast fungus races (Fig. $4)$. In the compatible interaction, WRKY45 began to be upregulated at 1.5 days postinoculation (dpi) and reached a peak of expression at $3 \mathrm{dpi}$, then declined to near the basal level by 5 dpi. In contrast, SalT expression began to increase at $3 \mathrm{dpi}$, and this upregulation continued until the end of the time course. On the other hand, the incompatible interaction caused only a minor induction of SalT expression, whereas it induced a much stronger and persistent expression of WRKY45 compared with that in the compatible interaction. Other ABA-respon sive genes, $O s W s i 18$ and $R A B 16 b$, showed expression patterns
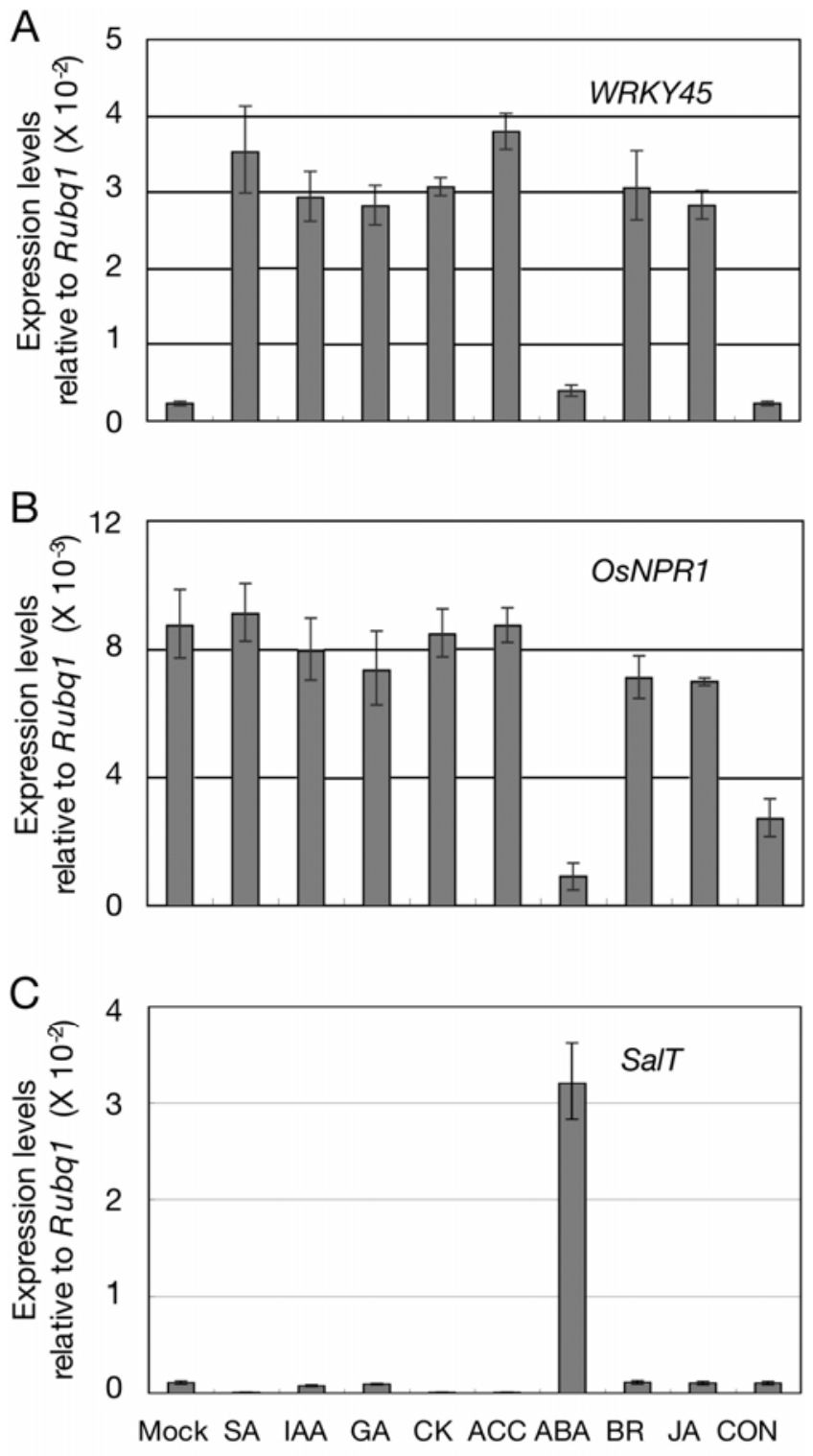

SA

Fig. 2. Abscisic acid (ABA) suppresses salicylic acid (SA)-induced upregulation of WRKY45 and OSNPR1. Leaf segments from rice seedlings were treated in solutions containing various plant hormones under light for $8 \mathrm{~h}$ at $30^{\circ} \mathrm{C}$. Transcript levels of A, WRKY45; $\mathbf{B}$, OsNPRI; and $\mathbf{C}$, SalT were determined by quantitative reverse-transcription polymerase chain reaction analyses using Rubq1 as an internal control. Mean values from three determinations with standard deviations are shown. SA (1 mM); IAA, indole-3acetic acid $(50 \mu \mathrm{M})$; GA, gibberellin $(50 \mu \mathrm{M})$; CK, kinetin $(50 \mu \mathrm{M})$; ACC, 1-aminocyclopropane-1-carboxylic acid $(50 \mu \mathrm{M})$; ABA $(50 \mu \mathrm{M}) ; \mathrm{BR}$, brassinolide $(2 \mu \mathrm{M})$; JA, jasmonic acid $(50 \mu \mathrm{M}) ; \mathrm{CON}$, leaf blades sampled directly from untreated rice seedlings. 


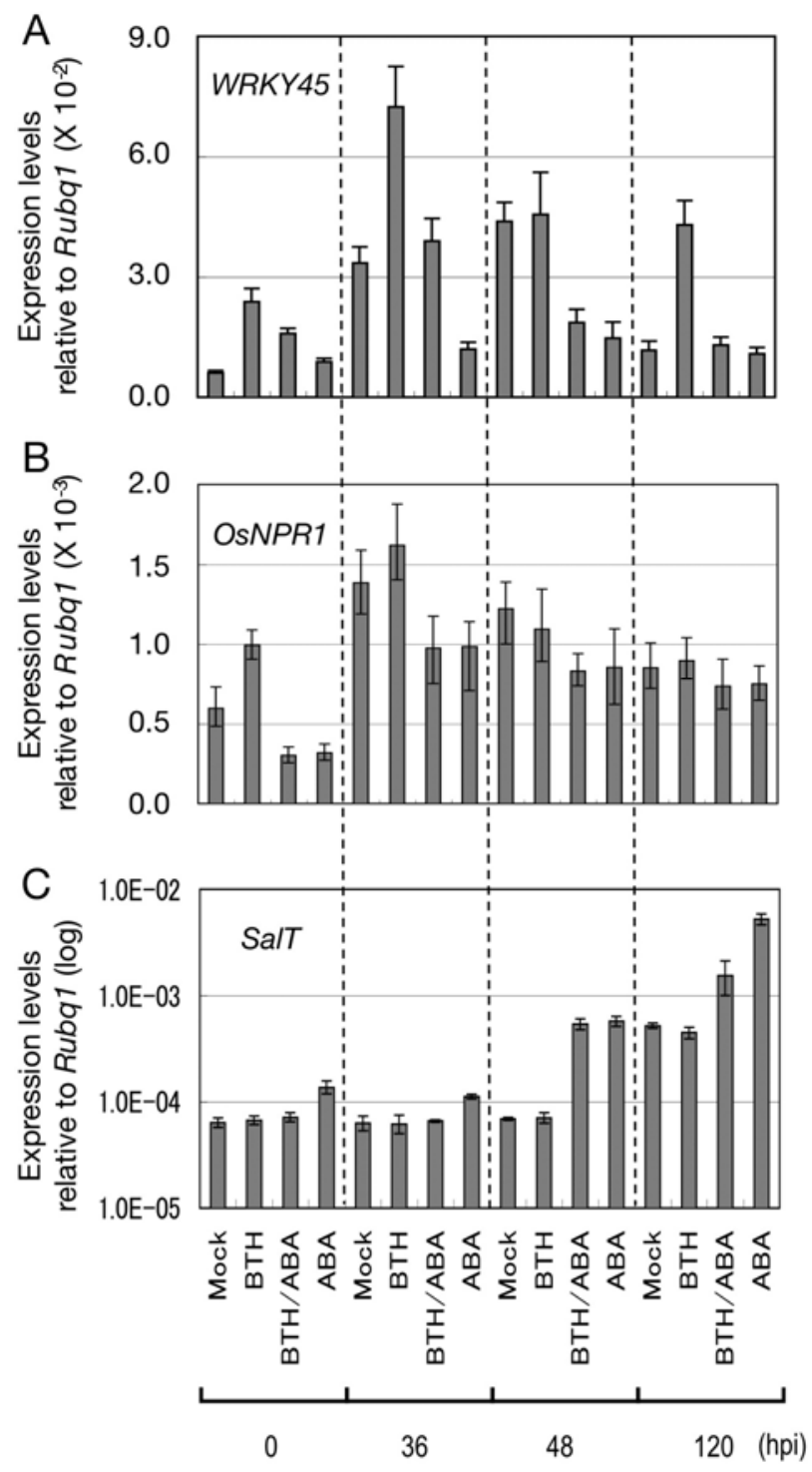

similar to that of SalT (Supplementary Fig. S5). OsPR1b showed upregulation concomitant with WRKY45, with higher expression levels observed during the incompatible interaction than the compatible one. These results strongly suggest the occurrence of a mutually antagonistic interaction between SA and ABA signaling pathways during blast infection, which is potentially important to the rice- $M$. grisea interaction.

\section{Overexpression of WRKY45 or OSNPR1 negates the effects of $\mathrm{ABA}$.}

Overexpression of WRKY45 in rice enhances rice resistance to $M$. grisea by strengthening the SA pathway (Shimono et al. 2007). To further validate the antagonistic interaction of ABA with the rice SA pathway and to determine the point at which ABA acted in the SA signaling pathway, we tested the effects of ABA application on the blast resistance of the transgenic lines overexpressing WRKY45. The plant seedlings were pretreated with ABA for $24 \mathrm{~h}$ by foliar spraying and then inoculated with compatible blast fungus (race 007.0). ABA application resulted in only a minor reduction in the blast resistance in the two transgenic rice lines overexpressing WRKY45 but did not restore blast susceptibility comparable with that of control wild-type plants (Fig. 5A). These results indicate that the negative impact of ABA on the SA-mediated defense signaling leading to the downregulation of WRKY45 was partially suppressed by WRKY45 overexpression. OsNPR1 also plays a key role in the rice SA pathway by regulating one of the branched pathways and its overexpression enhances rice resistance to $M$. grisea and $X$. oryzae (S. Sugano, unpublished data). Similar experiments using $O s N P R l$-overexpressing rice plants demonstrated that the negative impact of ABA on the OsNPR1 subpathway was also suppressed by $O S N P R 1$ overexpression. Collectively, these results further support the negative interaction of ABA on the rice SA pathway and that the action sites of ABA lie upstream of WRKY45 and OsNPR1.

\section{M. grisea produces and secretes ABA.}

The strong negative impact of ABA on the SA signaling pathway and its subsequent effects on blast resistance led us to

D

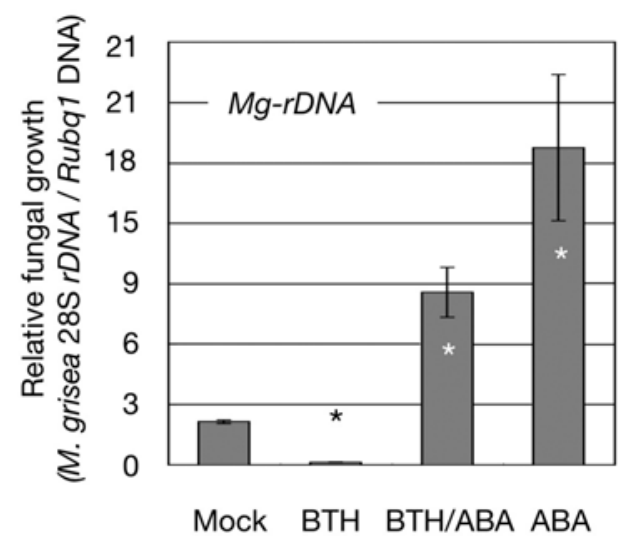

Fig. 3. Suppression of benzothiadiazole (BTH)-induced WRKY45/OsNPR1 expression by abscisic acid (ABA) and its effects on blast susceptibility. Rice seedlings at the four-leaf stage were pretreated for $24 \mathrm{~h}$ by spraying mock, BTH $(0.5 \mathrm{mM}), \mathrm{ABA}(0.1 \mathrm{mM})$, or $\mathrm{BTH}+\mathrm{ABA}$ solutions, and then inoculated with Magnaporthe grisea (race 007.0). Transcript levels of A, WRKY45; B, OsNPRI; and C, SalT in pretreated plants ( 0 h postinoculation [hpi]) and blast-inoculated plants at indicated time points (hpi) were determined by quantitative reverse-transcription polymerase chain reaction analyses using Rubq1 as an internal control. D, Severity of blast disease was evaluated by quantifying $M$. grisea genomic $28 \mathrm{~S}$ rDNA relative to rice genomic Rubq1 DNA (6 days postinoculation). Mean values of three determinations with standard deviations are shown. The asterisks indicate a significant difference from the mock control $(t$ test, $P<0.01)$.

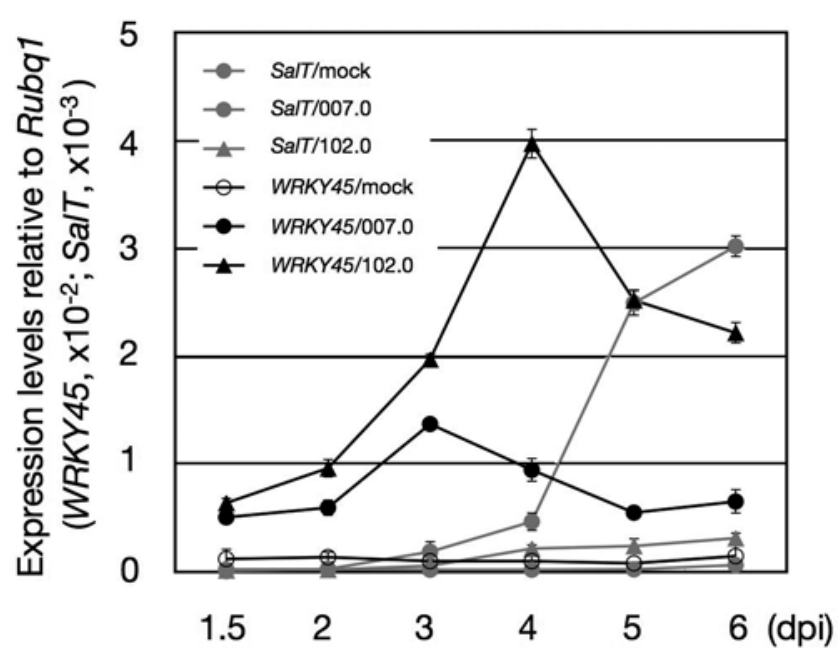

Fig. 4. Expression of marker genes for salicylic acid (SA) and abscisic acid (ABA) signaling pathways is inversely correlated during blast infection. Rice seedlings at the four-leaf stage were inoculated with compatible (race 007.0) or incompatible (race 102.0) blast fungus Magnaporthe grisea. Transcript levels of SA-responsive WRKY45 (solid lines) and ABA-responsive SalT (gray lines) genes were determined at the indicated time points (days postinoculation [dpi]) by quantitative reverse-transcription polymerase chain reaction analyses using Rubq1 as an internal control. Mean values of three determinations with standard deviations are shown. 
examine the possibility that $\mathrm{ABA}$ is derived from the fungus. Hormone analysis revealed that substantial amounts of ABA were accumulated in both hyphae (mycelium) and conidia, with higher levels detected in conidia compared with hyphae (Table 1). ABA was also detected in culture filtrates, indicating that the ABA produced in blast fungus was secreted into the culture medium. Whether fungus-derived $\mathrm{ABA}$ is responsible for the suppression of the rice SA pathway should be addressed in the future.

\section{DISCUSSION}

Rice blast disease, caused by the fungal pathogen $M$. grisea, is one of the most devastating diseases affecting cultivated rice that causes chronic yield losses and destabilizes annual production. It has often been observed that the incidence and severity of rice blast disease is influenced by environmental conditions. Generally, abiotic stresses, such as low temperature and drought, render rice plants more susceptible to blast disease (Bonman et al. 1988; Gill and Bonman 1988; Lai et al. 1999; Koga et al. 2004). Previous studies have suggested the possible linkage of ABA to this stress-associated rice susceptibility to blast disease (Kahn and Libby 1958; Kende et al. 1998; Lai et al. 1999; Saika et al. 2007; Bailey et al. 2009). In addition, exogenous application of ABA compromised rice resistance to $M$. grisea (Fig. 1) (Matsumoto et al. 1980; Koga et al. 2004; Bailey et al. 2009). On the other hand, ABA is also known to play a key role in plant responses to various abiotic stresses (Lee et al. 1995; Yamaguchi-Shinozaki and Shinozaki 2006). Hence, it appears that ABA plays an important regulatory role in the crosstalk between abiotic and biotic responses in rice to shift defense resources to the most life-threatening stress (Fujita et al. 2006; Asselbergh et al. 2008b).

The effects of ABA on plant defense pathways have been shown to be profound and drastic (Asselbergh et al. 2008b). In potato, for example, ABA pretreatment rendered potato slices vulnerable to infection by an incompatible isolate of Phytophthora infestans, and also even by the fungus Cladosporium cucumerinum that is normally nonpathogenic to potato (Henfling et al. 1980). It was also shown in soybean that ABA treatment compromised resistance to the incompatible isolates of $P$. sojae (McDonald and Cahill 1999). We showed that ABA treatment drastically enhanced disease susceptibility not only to compatible but also to incompatible blast fungus strains (Fig. 1). These results suggest that $\mathrm{ABA}$ negatively regulates both basal and $R$-gene-mediated defense pathways of rice plants to $M$. grisea. SA-mediated defense responses are an important part of both basal and $R$-gene-mediated resistance in Arabidopsis (Glazebrook 2001; Xiao et al. 2005; Venugopal et al. 2009). Therefore, it appears reasonable that ABA suppresses both types of resistance by suppressing the SA signaling pathway. The degrees of the effect of ABA on different incompatible rice-blast fungus combinations were considerably different (Fig. 1): ABA treatment resulted in severe blast disease symptoms in rice seedlings infected with blast fungus race 102.0 (Fig. 1C and D) but much lesser symptoms in those infected

Table 1. Abscisic acid (ABA) contents in hyphae and conidia of Magnaporthe grisea and culture medium

\begin{tabular}{lccc}
\hline & \multicolumn{3}{c}{ ABA (pmol per gram of fresh weight) } \\
\cline { 2 - 4 } Blast race & Hyphae & Filtrate & \multicolumn{1}{c}{ Conidia } \\
\hline 007.0 & $26.80 \pm 5.21$ & $2.76 \pm 0.69$ & $120.19 \pm 26.48$ \\
102.0 & $16.26 \pm 1.55$ & $1.61 \pm 0.37$ & $195.93 \pm \mathrm{nc}$ \\
\hline
\end{tabular}

${ }^{a}$ Values are represented as mean \pm standard deviation $(n=4) ; \mathrm{nc}=$ standard deviation was not calculated because there were only two samples. with race 002.0. Moreover, Koga and associates (2004) previously reported that, in an experiment using leaf sheaths, ABA treatment markedly suppressed the basal resistance of ricereferred to as whole-plant-specific resistance-in a compatible interaction, whereas no significant effect of ABA was observed in the incompatible rice (ZTR, $P i-z^{\prime}$ )-blast fungus (isolate Kita 1) interaction. In the light of our present study showing that ABA antagonizes the SA pathway, these data imply that different $R$-gene-mediated defenses depend upon the SA pathway to different extents. Alternatively, differences in the genetic background of fungal races used may be responsible for the observed difference.

In this study, we have shown that SA and ABA mutually antagonize each other's signaling pathways in rice. ABA suppressed SA (BTH)-induced expression of WRKY45 and
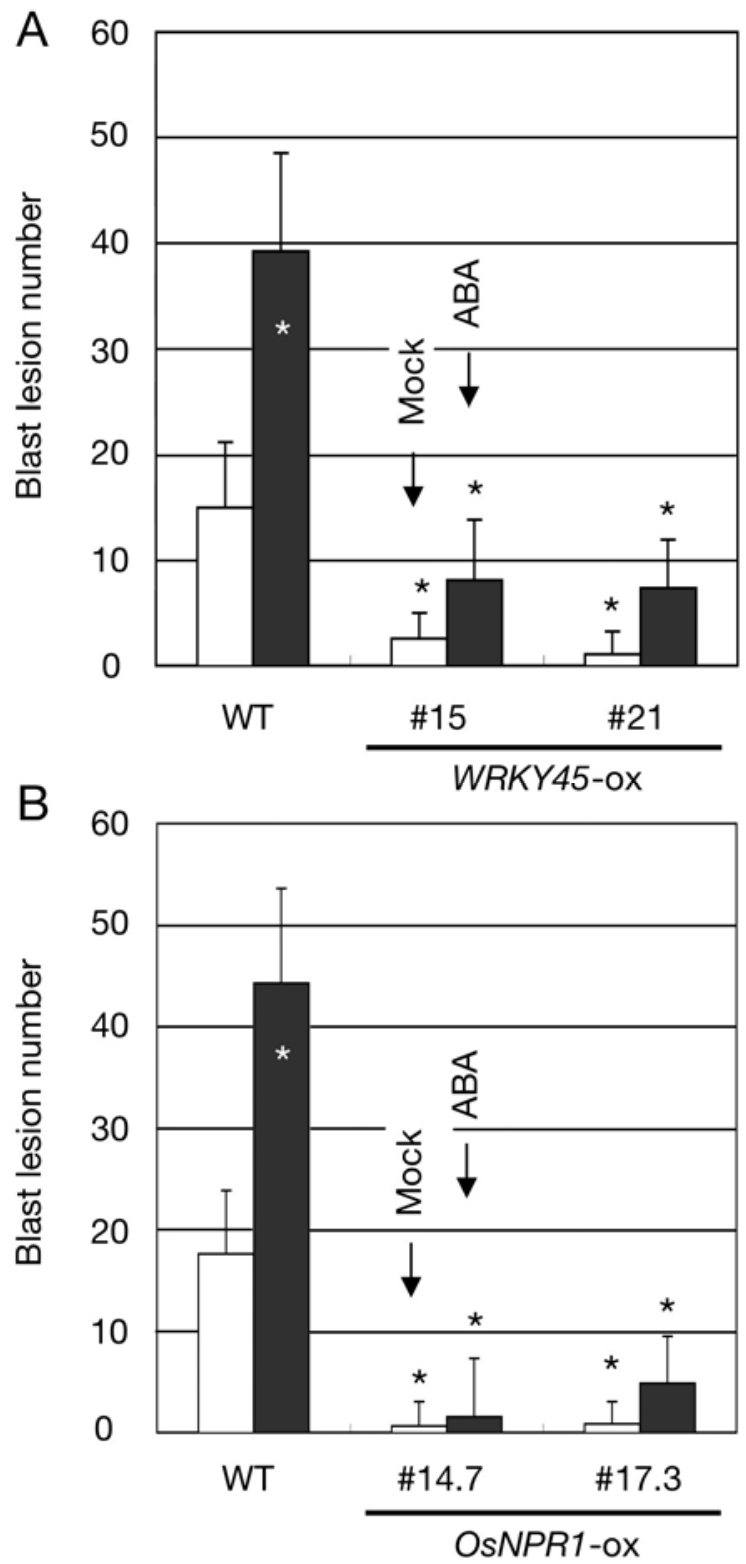

Fig. 5. Overexpression of $W R K Y 45$ or $O S N P R 1$ largely negates the abscisic acid (ABA)-enhanced susceptibility to Magnaporthe grisea. Control wild type (WT) and two lines each of A, WRKY45-ox and $\mathbf{B}, O S N P R 1$-ox transgenic rice plants at the four-leaf stage were sprayed with mock or ABA $(0.1 \mathrm{mM})$ solution and then inoculated with M. grisea (race 007.0) after 2 days. Mean values for the lesion number per $10-\mathrm{cm}$ middle region of leaf blades with standard deviations are shown. Asterisks indicate significant difference from the mock-treated WT plants $(t$ test, $P<0.01)$. 
OsNPR1, the two key components of the SA signaling pathway in rice, accompanied by an enhanced susceptibility to $M$. grisea infection (Figs. 2 and 3). Conversely, SA (BTH) suppressed ABA-responsive gene expression (Fig. 3). Overexpression of WRKY45 or OSNPR1 largely negated the negative impact of ABA on blast resistance (Fig. 5). We have also shown that the expression of marker genes for SA and ABA signaling pathways are inversely correlated during blast infection (Fig. 4). These data, taken together, strongly suggest that the balance of interaction between SA and ABA signaling is an important determinant for the outcome of rice- $M$. grisea interaction. The incompatible rice- $M$. grisea interaction induced only a minor upregulation of ABA-responsive genes, whereas it induced a much stronger expression of WRKY45 and $O s P R 1 b$ compared with the compatible interaction (Fig. 4). This suggests that the incompatible interaction strongly activates the SA pathway and causes the concomitant downregulation of the ABA pathway, resulting in strong blast resistance. The induction of ABA-responsive genes appeared to be preceded by WRKY45 expression during blast infection (Fig. 4). This may suggest that the antagonistic effects of ABA on the SA pathway act relatively later during blast infection, after transient activation of the SA pathway. However, we cannot rule out the possibility that early local activation of ABA signaling in host cells at the infection sites was missed because such local activation would be difficult to detect. Indeed, we found that ABA application later than $24 \mathrm{~h}$ after inoculation caused only a small increase in blast susceptibility (data not shown), implying that $\mathrm{ABA}$ action at earlier stages could be important. A transgenic rice line containing a reporter gene (e.g., $\beta$-glucuronidase) driven by an ABA-responsive promoter would aid in the detailed investigation of ABA signaling dynamics in the early stages of blast infection.

Recently, Bailey and associates (2009) have reported that the exogenous application of ABA drastically reduces ET levels in rice and enhances rice blast susceptibility. In addition, RNAi-

\section{M. grisea infection}
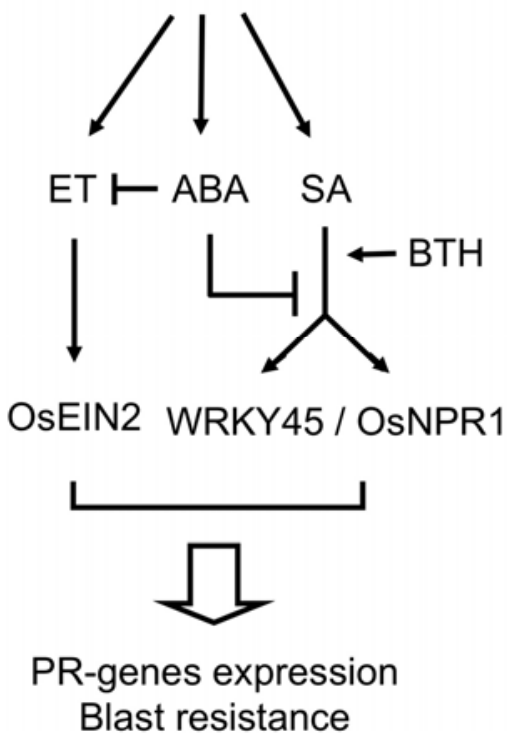

Fig. 6. Schematic illustration of salicylic acid (SA)-abscisic acid (ABA), and ethylene (ET)-ABA antagonistic crosstalks in rice-Magnaporthe grisea interaction. In response to the pathogen infection ( $M$. grisea), rice plants activate SA and ET signaling pathways, upon which ABA acts negatively. $M$. grisea may utilize this antagonism of signaling pathways as a means to slip through the rice defense mechanism, possibly by using its own ABA. Arrows and lines with a bar at the end indicate positive and negative regulation, respectively. mediated suppression of OsEIN2b-a central component of ET signal transduction-resulted in ABA-hypersensitivity, reduced defense gene expression, and enhanced blast susceptibility. These data suggest that ABA antagonistically interacts with the ET signaling pathway and, consequently, enhances susceptibility of rice to $M$. grisea. Taken together, it appears that ABA opposes both SA and ET signaling pathways to suppress the defense response against $M$. grisea in rice (Fig. 6).

Many fungal and bacterial phytopathogens have developed various mechanisms to interfere with hormonal balances in host plants for efficient infection or growth, either by producing hormones by themselves or by modulating hormone synthesis pathways in host plants (Robert-Seilaniantz et al. 2007; Grant and Jones 2009). For example, Pseudomonas syringae secretes T3Es to induce ABA synthesis in planta during the interaction with Arabidopsis, thereby suppressing SA production and the basal defense of the host plants (de Torres-Zabala et al. 2007). Several fungal pathogens, such as Botrytis cinerea, Ceratocystis coerulescens, Fusarium oxysporum, and Rhizoctonia solani, can themselves produce ABA (Dörffling et al. 1984; Kettner and Dörffling 1987; Oritani and Kiyota 2003), although the relevance of the ABA production of these pathogens to their pathogenicity remains unknown. In this study, the blast fungus $M$. grisea and its culture media were found to contain ABA (Table 1), indicating that the fungus produces and secretes ABA. These results imply that the fungus may use its own ABA to trigger ABA signaling at host infection sites such that it suppresses SA and ET signaling pathways to alleviate rice defense responses.

\section{MATERIALS AND METHODS}

Plant materials and growth conditions.

The Japonica rice Oryza sativa subsp. japonica cv. Nipponbare was grown in a greenhouse on soil (Bonsol No. 2; Sumitomo Chemical Corp., Tokyo) at $28^{\circ} \mathrm{C}$ in the day and $23^{\circ} \mathrm{C}$ at night. The relative humidity in the greenhouse was approximately $70 \%$.

\section{Chemical treatments.}

All stock solutions, except brassinolide (BR), were prepared at a concentration of $100 \mathrm{mM}$ as described previously (Jiang et al. 2008). BR was prepared at $20 \mathrm{mM}$ concentration. Indole-3acetic acid (IAA) (Sigma-Aldrich, St. Louis), gibberellin A3 $\left(\mathrm{GA}_{3}\right)$ (Wako, Osaka, Japan), 1-aminocyclopropane-1-carboxylic acid (ACC), ABA ([ \pm$]$-cis-trans) (Sigma-Aldrich), methyl jasmonate (ME-JA) (Wako), and (BR) (Wako) were dissolved in ethanol. Kinetin (CK) (Sigma-Aldrich) and BTH $S$-methyl ester (Wako) were dissolved in dimethyl sulfoxide (DMSO); and ethephon (ET) (Sigma-Aldrich) and SA (Nacalai Tesque, Tokyo) in $\mathrm{H}_{2} \mathrm{O}$. The solvents did not exceed a final concentration of $0.1 \%$ in the solutions used for plant treatments and had no effect on the rice gene expressions examined in this work.

Leaf blades from rice plants at the four-leaf stage were cut into segments of approximately $0.5 \mathrm{~cm}$ in length and submerged in a solution containing the chemicals prepared in $0.01 \%$ Silwet L-77. The leaf segments were then incubated under light for 8 $\mathrm{h}$ at $30^{\circ} \mathrm{C}$. In some experiments, rice plants at the four-leaf-stage were foliar sprayed with a chemical solution prepared in $0.02 \%$ Silwet L-77.

\section{RNA analyses.}

Total RNA was isolated from leaf blades of the fourth leaf of rice seedlings using the Trizol reagent (Invitrogen, Carlsbad, CA, U.S.A.). Quantitative reverse-transcription polymerase chain reaction (qRT-PCR) was run on a Thermal Cycler Dice TP800 system (Takara Bio, Tokyo) using SYBR premix Ex 
Taq mixture (Takara Bio) as described previously (Shimono et al. 2007). Primer sequences used for qRT-PCR are listed in Supplementary Table 1.

\section{Pathogen culture and inoculations.}

Culture and inoculations of the blast fungus $M$. grisea (compatible race 007.0 and incompatible races 102.0 and 002.0) were carried out essentially as described previously (Jiang et al. 2009), with slight modifications. Briefly, the fungus was grown on an oatmeal agar medium (oatmeal, 30 $\mathrm{g} /$ liter; sucrose, $5 \mathrm{~g} /$ liter; and agar, $16 \mathrm{~g} /$ liter) at $26^{\circ} \mathrm{C}$ for 10 to 12 days. After removing the aerial hyphae by washing with distilled water and a brush, conidia formation was induced by irradiation under continuous black blue light (FL15BLB; Toshiba, Osaka, Japan) at $24^{\circ} \mathrm{C}$ for 3 days. The conidia were suspended in $0.02 \%$ Silwet L-77 at a density of $10^{5} / \mathrm{ml}$ and sprayed onto rice plants at the four-leaf stage. After incubation in a dew chamber at $24^{\circ} \mathrm{C}$ for $24 \mathrm{~h}$, the rice plants were moved back to the greenhouse.

Disease development was evaluated either by counting the number of blast lesions per $10-\mathrm{cm}$ middle region of each fourth leaf or by determining $M$. grisea genomic 28S rDNA (Qi and Yang 2002) by qRT-PCR (Shimono et al. 2007) at 6 to 7 days after inoculation. At least 20 plants were used for each disease assay.

\section{Measurement of ABA content.}

For determination of ABA, M. grisea (race 007.0 and 102.0) was grown in $50 \mathrm{ml}$ of $5 \times$ YEG medium $(0.5 \%$ yeast extract, $1 \%$ glucose) at $25^{\circ} \mathrm{C}$ on a rotary shaker for 7 days. The culture was filtered through Miracloth (Calbiochem, La Jolla, CA, U.S.A.) and the mycelium was frozen in liquid nitrogen and homogenized. The culture filtrate was centrifuged at $2,000 \times g$ for $10 \mathrm{~min}$ at room temperature to remove the mycelia completely, and the resulting supernatant was frozen in liquid nitrogen and lyophilized. Conidia were induced on an oatmeal agar medium and suspended in $0.02 \%$ Silwet L-77 as described above, and then, they were collected in a 2-ml Eppendorf tube by a brief centrifugation. The conidia were then frozen in liquid nitrogen and homogenized. The homogenates of mycelium and conidia and the culture filtrate were subjected to ABA determination according to the procedures described previously (Kojima et al. 2009).

\section{ACKNOWLEDGMENTS}

We thank H. Onodera for her technical support in generating rice transformants. This work was supported by a grant from the Japanese Ministry of Agriculture, Forestry, and Fisheries (Genomics for Agricultural Innovation, GMA0001 and PMI0008).

\section{LITERATURE CITED}

Achuo, E. A., Prinsen, E., and Höfte, M. 2006. Influence of drought, salt stress and abscisic acid on the resistance of tomato to Botrytis cinerea and Oidium neolycopersici. Plant Pathol. 55:178-186.

Asselbergh, B., Curvers, K., Franca, S. C., Audenaert, K., Vuylsteke, M., Van Breusegem, F., and Hofte, M. 2007. Resistance to Botrytis cinerea in sitiens, an abscisic acid-deficient tomato mutant, involves timely production of hydrogen peroxide and cell wall modifications in the epidermis. Plant Physiol. 144:1863-1877.

Asselbergh, B., Achuo, A. E., Hofte, M., and Van Gijsegem, F. 2008a. Abscisic acid deficiency leads to rapid activation of tomato defence responses upon infection with Erwinia chrysanthemi. Mol. Plant Pathol. 9:11-24.

Asselbergh, B., De Vleesschauwer, D., and Hofte, M. 2008b. Global switches and fine-tuning-ABA modulates plant pathogen defense. Mol. Plant-Microbe Interact. 21:709-719.

Audenaert, K., De Meyer, G. B., and Hofte, M. M. 2002. Abscisic acid determines basal susceptibility of tomato to Botrytis cinerea and sup- presses salicylic acid-dependent signaling mechanisms. Plant Physiol. 128:491-501.

Bailey, T., Zhou, X., Chen, J., and Yang, Y. 2009. Role of ethylene, abscisic acid and MAP kinase pathways in rice blast resistance. Pages 185-190 in: Advances in Genetics, Genomics and Control of Rice Blast Disease. G.-L. W. and B. Valent, eds. Springer, The Netherlands.

Bonman, J. M., Sanchez, L. M., and Mackill, A. O. 1988. Effects of water deficit on rice blast. II. Disease-development in the field. J. Plant Prot. Trop. 5:67-73.

Brooks, D. B., Bender, C. L., and Kunkel, B. N. 2005. The Pseudomonas syringae phytotoxin coronatine promotes virulence by overcoming salicylic acid-dependent defences in Arabidopsis thaliana. Mol. Plant Pathol. 6:629-639.

Chern, M. S., Fitzgerald, H. A., Yadav, R. C., Canlas, P. E., Dong, X., and Ronald, P. C. 2001. Evidence for a disease-resistance pathway in rice similar to the NPR1-mediated signaling pathway in Arabidopsis. Plant J. 27:101-113.

Chern, M., Fitzgerald, H. A., Canlas, P. E., Navarre, D. A., and Ronald, P. C. 2005. Overexpression of a rice NPR1 homolog leads to constitutive activation of defense response and hypersensitivity to light. Mol. PlantMicrobe Interact. 18:511-520.

de Torres-Zabala, M., Truman, W., Bennett, M. H., Lafforgue, G., Mansfield, J. W., Rodriguez Egea, P., Bogre, L., and Grant, M. 2007. Pseudomonas syringae pv. tomato hijacks the Arabidopsis abscisic acid signalling pathway to cause disease. EMBO (Eur. Mold. Biol. Organ.) J. 26:1434-1443.

de Torres Zabala, M., Bennett, M. H., Truman, W. H., and Grant, M. R. 2009. Antagonism between salicylic and abscisic acid reflects early host-pathogen conflict and moulds plant defence responses. Plant J. 59:375-386.

Dong, X. 2004. NPR1, all things considered. Curr. Opin. Plant Biol. 7:547-552.

Dörffling, K., Petersen, W., Sprecher, E., Urbasch, I., and Hanssen, H. P. 1984. Abscisic acid in phytopathogenic fungi of the genera Botrytis, Ceratocystis, Fusarium, and Rhizoctonia. Z. Naturforsch. 39:683-684.

Durrant, W. E., and Dong, X. 2004. Systemic acquired resistance. Annu. Rev. Phytopathol. 42:185-209.

Fan, J., Hill, L., Crooks, C., Doerner, P., and Lamb, C. 2009. Abscisic acid has a key role in modulating diverse plant-pathogen interactions. Plant Physiol. 150:1750-1761.

Fitzgerald, H. A., Chern, M. S., Navarre, R., and Ronald, P. C. 2004. Overexpression of (At)NPR1 in rice leads to a BTH- and environment-induced lesion-mimic/cell death phenotype. Mol. Plant-Microbe Interact. 17:140-151.

Flors, V., Ton, J., van Doorn, R., Jakab, G., Garcia-Agustin, P., and Mauch-Mani, B. 2008. Interplay between JA, SA and ABA signalling during basal and induced resistance against Pseudomonas syringae and Alternaria brassicicola. Plant J. 54:81-92.

Fujita, M., Fujita, Y., Noutoshi, Y., Takahashi, F., Narusaka, Y., YamaguchiShinozaki, K., and Shinozaki, K. 2006. Crosstalk between abiotic and biotic stress responses: a current view from the points of convergence in the stress signaling networks. Curr. Opin. Plant Biol. 9:436-442.

Gill, M. A., and Bonman, J. M. 1988. Effects of water deficit on rice blast. I. Influence of water deficit on components of resistance. J. Plant Prot. Trop. 5:61-66.

Glazebrook, J. 2001. Genes controlling expression of defense responses in Arabidopsis-2001 status. Curr. Opin. Plant Biol. 4:301-308.

Grant, M. R., and Jones, J. D. 2009. Hormone (dis)harmony moulds plant health and disease. Science 324:750-752.

Hayashi, N., Ando, I., and Imbe, T. 1998. Identification of a new resistance gene to a Chinese blast fungus isolate in the Japanese rice cultivar Aichi Asahi. Phytopathology 88:822-827.

Henfling, J., Bostock, R., and Kuc, J. 1980. Effect of abscisic acid on rishitin and lubimin accumulation and resistance to Phytophthora infestans and Cladosporium cucumerinum in potato tuber tissue slices. Phytopathology 70:1074-1078.

Imbe, T., and Matsumoto, S. 1985. Inheritance of resistance of rice varieties to blast fungus strains virulent to the variety "Reiiho". Jpn. J. Breed. 35:332-339.

Jiang, C. J., Aono, M., Tamaoki, M., Maeda, S., Sugano, S., Mori, M., and Takatsuji, H. 2008. SAZ, a new SUPERMAN-like protein, negatively regulates a subset of ABA-responsive genes in Arabidopsis. Mol. Genet. Genomics 279:183-192.

Jiang, C. J., Shimono, M., Maeda, S., Inoue, H., Mori, M., Hasegawa, M., Sugano, S., and Takatsuji, H. 2009. Suppression of the rice fatty-acid desaturase gene OsSSI2 enhances resistance to blast and leaf blight diseases in rice. Mol. Plant-Microbe Interact. 22:820-829.

Joshee, N., Kisaka, H., and Kitagawa, Y. 1998. Isolation and characterization of a water stress-specific genomic gene, pwsi18, from rice. Plant Cell Physiol. 39:64-72. 
Kahn, R. P., and Libby, J. L. 1958. The effect of environmental factors and plant ages on the infection of rice by the blast fungus, Pyricularia oryzae. Phytopathology 48:25-30.

Kende, H., van der Knaap, E., and Cho, H. T. 1998. Deepwater rice: a model plant to study stem elongation. Plant Physiol. 118:1105-1110.

Kettner, J., and Dörffling, K. 1987. Abscisic acid metabolism in Ceratocystis coerulescens. Physiol. Plant 69:278-282.

Koga, H., Dohi, K., and Mori, M. 2004. Abscisic acid and low temperatures suppress the whole plant-specific resistance reaction of rice plants to the infection of Magnaporthe grisea. Physiol. Mol. Plant Pathol. 65:3-9.

Kojima, M., Kamada-Nobusada, T., Komatsu, H., Takei, K., Kuroha, T., Mizutani, M., Ashikari, M., Ueguchi-Tanaka, M., Matsuoka, M., Suzuki, K., and Sakakibara, H. 2009. Highly sensitive and highthroughput analysis of plant hormones using MS-probe modification and liquid chromatography-tandem mass spectrometry: an application for hormone profiling in Oryza sativa. Plant Cell Physiol. 50:12011214.

Lai, X. H., Marchetti, M. A., and Petersen, H. D. 1999. Comparative slowblasting in rice grown under upland and flooded blast nursery culture. Plant Dis. 93:681-684.

Laurie-Berry, N., Joardar, V., Street, I. H., and Kunkel, B. N. 2006. The Arabidopsis thaliana JASMONATE INSENSITIVE 1 gene is required for suppression of salicylic acid-dependent defenses during infection by Pseudomonas syringae. Mol. Plant-Microbe Interact. 19:789-800.

Lee, T.-M., Lur, H.-S., and Chu, C. 1995. Role of abscisic acid in chilling tolerance of rice (Oryza sativa L.) seedlings. I. Endogenous abscisic acid levels. Plant Cell Environ. 16:481-490.

Loake, G., and Grant, M. 2007. Salicylic acid in plant defence-the players and protagonists. Curr. Opin. Plant Biol. 10:466-472.

Lopez, M. A., Bannenberg, G., and Castresana, C. 2008. Controlling hormone signaling is a plant and pathogen challenge for growth and survival. Curr. Opin. Plant Biol. 11:420-427.

Matsumoto, K., Suzuki, Y., Mase, S., Watanabe, T., and Sekizawa, Y. 1980. On the relationship between plant hormones and rice blast resistance. Ann. Phytopathol. Soc. Jpn. 46:307-314.

Mauch-Mani, B., and Mauch, F. 2005. The role of abscisic acid in plantpathogen interactions. Curr. Opin. Plant Biol. 8:409-414.

McDonald, K., and Cahill, D. 1999. Influence of abscisic acid and the abscisic acid biosynthesis inhibitor, norflurazon, on interactions between Phytophthora sojae and soybean (Glycine max). Eur. J. Plant Pathol. 105:651-658

Melotto, M., Underwood, W., Koczan, J., Nomura, K., and He, S. Y. 2006. Plant stomata function in innate immunity against bacterial invasion. Cell 126:969-980.

Mohr, P. G., and Cahill, D. M. 2003. Abscisic acid influences the susceptibility of Arabidopsis thaliana to Pseudomonas syringae pv. tomato and Peronospora parasitica. Funct. Plant Biol. 30:461-469.

Oritani, T., and Kiyota, H. 2003. Biosynthesis and metabolism of abscisic acid and related compounds. Nat. Prod. Rep. 20:414-425.

Qi, M., and Yang, Y. 2002. Quantification of Magnaporthe grisea during infection of rice plants using real-time polymerase chain reaction and northern blot/phosphoimaging analyses. Phytopathology 92:870-876.

Qiu, Y., and Yu, D. 2009. Over-expression of the stress-induced OsWRKY45 enhances disease resistance and drought tolerance in Arabidopsis. Environ. Exp. Bot. 65:35-47.

Rabbani, M. A., Maruyama, K., Abe, H., Khan, M. A., Katsura, K., Ito,
Y., Yoshiwara, K., Seki, M., Shinozaki, K. and, Yamaguchi-Shinozaki, K. 2003. Monitoring expression profiles of rice genes under cold, drought, and high-salinity stresses and abscisic acid application using cDNA microarray and RNA gel-blot analyses. Plant Physiol. 133:17551767.

Ribot, C., Hirsch, J., Balzergue, S., Tharreau, D., Notteghem, J. L. Lebrun, M. H., and Morel, J. B. 2008. Susceptibility of rice to the blast fungus, Magnaporthe grisea. J. Plant Physiol. 165:114-124.

Robert-Seilaniantz, A., Navarro, L., Bari, R., and Jones, J. D. 2007. Pathological hormone imbalances. Curr. Opin. Plant Biol. 10:372-379.

Saika, H., Okamoto, M., Miyoshi, K., Kushiro, T., Shinoda, S., Jikumaru, Y., Fujimoto, M., Arikawa, T., Takahashi, H., Ando, M., Arimura, S., Miyao, A., Hirochika, H., Kamiya, Y., Tsutsumi, N., Nambara, E., and Nakazono, M. 2007. Ethylene promotes submergence-induced expression of OsABA8ox1, a gene that encodes ABA 8'-hydroxylase in rice. Plant Cell Physiol. 48:287-298.

Shimono, M., Sugano, S., Nakayama, A., Jiang, C. J., Ono, K., Toki, S., and Takatsuji, H. 2007. Rice WRKY45 plays a crucial role in benzothiadiazole-inducible blast resistance. Plant Cell 19:2064-2076.

Ton, J., and Mauch-Mani, B. 2004. Beta-amino-butyric acid-induced resistance against necrotrophic pathogens is based on ABA-dependent priming for callose. Plant J. 38:119-130.

Vallad, G. E., and Goodman, R. M. 2004. Systemic acquired resistance and induced systemic resistance in conventional agriculture. Crop Sci. 44:1920-1934

Venugopal, S. C., Jeong, R. D., Mandal, M. K., Zhu, S., Chandra-Shekara, A. C., Xia, Y., Hersh, M., Stromberg, A. J., Navarre, D., Kachroo, A., and Kachroo, P. 2009. Enhanced disease susceptibility 1 and salicylic acid act redundantly to regulate resistance gene-mediated signaling. PLoS Genet. 5:e1000545.

Wang, D., Amornsiripanitch, N., and Dong, X. 2006. A genomic approach to identify regulatory nodes in the transcriptional network of systemic acquired resistance in plants. PLoS Pathog. 2:e123.

Ward, E. W., Cahill, D. M., and Bhattacharyya, M. K. 1989. Abscisic acid suppression of phenylalanine ammonia-lyase activity and mRNA, and resistance of soybeans to Phytophthora megasperma f. sp. glycinea. Plant Physiol. 91:23-27.

Xiao, S., Calis, O., Patrick, E., Zhang, G., Charoenwattana, P., Muskett, P. Parker, J. E., and Turner, J. G. 2005. The atypical resistance gene, $R P W 8$, recruits components of basal defence for powdery mildew resistance in Arabidopsis. Plant J. 42:95-110.

Yamaguchi-Shinozaki, K., and Shinozaki, K. 2006. Transcriptional regulatory networks in cellular responses and tolerance to dehydration and cold stresses. Annu. Rev. Plant Biol. 57:781-803.

Yamaguchi-Shinozaki, K., Mundy, J., and Chua, N.-H. 1989. Four tightly linked $r a b$ genes are differentially expressed in rice. Plant Mol. Biol. 14:29-39.

Yasuda, M., Ishikawa, A., Jikumaru, Y., Seki, M., Umezawa, T., Asami, T. Maruyama-Nakashita, A., Kudo, T., Shinozaki, K., Yoshida, S., and Nakashita, H. 2008. Antagonistic interaction between systemic acquired resistance and the abscisic acid-mediated abiotic stress response in Arabidopsis. Plant Cell 20:1678-1692.

Yuan, Y., Zhong, S., Li, Q., Zhu, Z., Lou, Y., Wang, L., Wang, J., Wang, M., Li, Q., Yang, D., and He, Z. 2007. Functional analysis of rice NPR1-like genes reveals that OsNPR1/NH1 is the rice orthologue conferring disease resistance with enhanced herbivore susceptibility. Plant Biotechnol. J. 5:313-324. 